\title{
Stereotactic radiosurgery for treatment of large cerebellum metastases from lung cancer
}

\author{
Mingyao Lai", Shaoqun Li", Jiangfen Zhou, Junjie Zhen, Juan Li, Qingjun Hu, Changguo Shan, \\ Ruyu Ai, Weiping Hong, Hui Wang, Minting Ye, Yanying Yang, Xiao Xiao, Lei Wen, Zhaoming Zhou, \\ Cheng Zhou, Linbo Cai^
}

Department of Neuro-Oncology, Guangdong Sanjiu Brain Hospital, Guangzhou, China

Contributions: (I) Conception and design: L Cai; (II) Administrative support: L Cai; (III) Provision of study materials or patients: All authors; (IV) Collection and assembly of data: All authors; (V) Data analysis and interpretation: M Lai, S Li; (VI) Manuscript writing: All authors; (VII) Final approval of manuscript: All authors.

"These authors contributed equally to this work.

Correspondence to: Linbo Cai. Department of Neuro-Oncology, Guangdong Sanjiu Brain Hospital, No. 578, South ShaTai Road, Guangzhou 510515,

China. Email: cailinbo999@163.com.

Background Stereotactic radiosurgery (SRS) is an important treatment option. This report evaluated the efficacy and safety of SRS in patients with large cerebellum metastases from lung cancer.

Methods: Between September 2016 and January 2020, a total of 44 patients with large cerebellum metastases $>2 \mathrm{~cm}$ from lung cancer were evaluated. A median dose of 20 Gy (range, 8-24 Gy) was delivered in 1 to 3 fractions for SRS treatment. The survival rate was analyzed with SPSS software 21.0 and compared by log-rank test using the Kaplan-Meier method.

Results: The median overall survival (OS) and neurological progression-free survival (PFS) were 10.5 months (range, 1-32 months) and 9.0 months (range, 1-32 months), respectively. The median diameter and volume of the metastases were $3.5 \mathrm{~cm}$ (range, 2.1-5.7 cm) and $12.5 \mathrm{cc}$ (range, 1.8-39.7 cc), respectively. The median volume of peritumoral edema was $36.3 \mathrm{cc}$ (range, 3.7-100.3 cc). The median ratio of tumor volume to cerebellum volume was $8.7 \%$ (range, 1.3-27.0\%). The median ratio of peritumoral edema volume to cerebellum volume was $25.0 \%$ (range, 2.5-68.6\%). Neurological symptoms were present in $97.7 \%(43 / 44)$ of patients. After SRS treatment, symptoms improved in $83.7 \%(36 / 44)$ patients, stabilized in $11.6 \%(5 / 44)$ patients, whilst two patients experienced symptomatic progression. Of the latter, one patient accepted emergency surgery and the other accepted palliative care.

Conclusions: Large cerebellum metastases are amongst the most severe forms of brain tumors. Increased tumor volume and peritumoral edema volume correlate with the most severe symptoms. SRS may be an effective alternative treatment for large cerebellum metastases from lung cancer and may preserve neurological function.

Keywords: Stereotactic radiosurgery (SRS); large cerebellum metastases; lung cancer

Submitted Sep 24, 2020. Accepted for publication Dec 26, 2020.

doi: 10.21037/apm-20-2237

View this article at: http://dx.doi.org/10.21037/apm-20-2237

^ORCID: 0000-0003-1011-9868. 


\section{Introduction}

Brain metastases are the most common type of intracranial tumor, the incidence of which is increasing due to the improved outcome of systemic therapy and the now routine use of brain magnetic resonance imaging (MRI) screening in detecting tumors. The management of brain metastases is determined by factors such as the number, location and size of tumors, the volume of peritumoral edema, and patient factors such as primary diseases factors and patient age and performance status. A small number of studies have demonstrated single-session and multisessionsession stereotactic radiosurgery (SRS) to be effective and safe in treating large brain metastases (1-4), however, clinical radiation oncologists are cautious about the use of SRS in their treatment. Although large cerebellum lesions are often amenable to surgical resection, neurological function is difficult to restore. Cerebrotomy may also cause wound complications and leptomeningeal spreading, especially in the posterior fossa, and delay in establishing further systemic oncological therapies. SRS may enlarge peritumoral edema in the cerebellum, leading to severe neurological complications.

Most studies to date have focused on the diameter or volume of the lesion in defining the size of brain metastases. Lesions with a diameter $>2$ or $3 \mathrm{~cm}$, or volume $>10$ or 15 cc, have been defined as large in different studies $(2,5-7)$, however there appears to be no standard definition. Whilst these studies have paid close attention to tumor size and volume, the volume and extent of peritumoral edema has, however, been ignored, in spite of this having a significant impact on the status of patients and increasing the risk of adverse effects from SRS.

In this study, we evaluate the efficacy and safety of SRS in patients with large cerebellum metastases from lung cancer. As space in the posterior fossa is limited, we define a tumor as large if it exceeds $2 \mathrm{~cm}$ in diameter. The study also evaluates clinical features, tumor volume and peritumoral edema volume in these patients.

We present the following article in accordance with the STROBE reporting checklist (available at http://dx.doi. org/10.21037/apm-20-2237).

\section{Methods}

\section{Inclusion criteria and exclusion criteria}

Between September 2016 and January 2020, a total of 44 patients with cerebellum metastases from lung cancer were evaluated according to the inclusion and exclusion criteria as follows: inclusion criteria, (I) age $>18$ years, with histologically proven lung cancer; (II) cerebellum metastases with a diameter $>2 \mathrm{~cm}$; (III) receiving SRS treatment. Exclusion criteria: (I) raised severe intracranial pressure for which drugs were ineffective; (II) pregnant or breastfeeding patients.

The study was approved by IRB of Guangdong Sanjiu Brain Hospital (No. 2020-020-083) and written informed consent was obtained from all patients. All procedures performed in this study involving human participants were in accordance with the Declaration of Helsinki (as revised in 2013).

\section{SRS}

SRS was performed by using iPlan 4.0 treatment planning system (BrainLAB, Heimstetten, Germany) or Varian Eclipse treatment planning systems (Varian Medical Systems, Palo Alto, USA). Patients were immobilized with a thermoplastic mash mask. Treatment planning CT images were acquired with a $1.5 \mathrm{~mm}$ slice thickness. Brain MRI including $5.5 \mathrm{~mm}$ slices axial/sagittal/coronal T2 FLAIR-weighted sequences, $2 \mathrm{~mm}$ slices axial T2 FLAIRweighted sequences, $5.5 \mathrm{~mm}$ slices axial/sagittal/coronal T1-weighted gadolinium enhanced sequences and $2 \mathrm{~mm}$ slices axial T1-weighted gadolinium enhanced sequences were also obtained. The MRIs images were registered and automatically fused with the planning CT images in the treatment-planning system to facilitate the delineation of the tumor volume (equal to the planning target volume). The critical organ structures including the brainstem, the eyes, the lens, the optic nerves and the chiasm would be delineated. Generally, radiation dose was selected based on the recommendations from the trial Radiation Therapy Oncology Group (RTOG) 90-05, with modifications based on individual cases and considering tumor size, location, and patient status. We prescribed doses of 16-18 Gy in single session or 6-12 Gy in two or three daily fractions via isocenter plans.

\section{Follow-up and statistical analyses}

After SRS treatment, each patient was followed up with neurological and radiological examinations. Follow-up MRI scans were obtained routinely at 1 to 3 months after treatment or in the event of neurological deterioration. The first follow-up brain MRI was reviewed to assess the post-SRS condition of the brain lesion and its relation to 
neurological symptoms. Treatment response assessments were based on the Response Assessment in NeuroOncology for brain metastases (the RANO-BM criteria) (8).

Overall survival (OS) and neurological progressionfree survival (PFS) were estimated using the KaplanMeier method calculated from the date of treatment start to the date of events or the last follow-up. Factors possibly affecting the survival were tested using the log-rank test for univariate analysis and the Cox proportional hazards models with variable selection, which included age, gender, smoking, symptom, tumor location, numbers of tumor, status of primary cancer, presence of extracranial metastases, pretreatment KPS score, Radiation Therapy Oncology Group-recursive partitioning analysis (ROTG-RPA) class, graded prognostic assessment (GPA) score, hemorrhage, hydrocephalus and systemic therapy. All statistical tests were conducted using SPSS version 21.0. Statistical significance was set at $\mathrm{P}<0.05$.

\section{Results}

\section{Characteristics of patients}

The baseline characteristics of this study are summarized in Table 1. Of the 44 patients, $30(68.2 \%)$ were $>50$ years old and $14(31.8 \%)$ were $\leq 50$ years old. There were $29(65.9 \%)$ male patients and $15(34.1 \%)$ female. Only 1 patient was asymptomatic, their tumor being detected during a routine check, whilst $43(97.7 \%)$ were symptomatic. The KPS score was $\geq 70$ in 35 patients $(79.5 \%)$, and adenocarcinoma was present in 26 patients $(59.1 \%)$. The ROTG-RPA classification saw 5 patients $(11.4 \%)$ designated as class I, 32 patients $(72.7 \%)$ as class II, and $7(15.9 \%)$ as class III. The GPA score was $\leq 1$ in 10 patients (22.7\%), 1.5-2.5 in 21 patients $(47.7 \%)$ and $\geq 3$ in 13 patients $(29.5 \%)$. The primary cancer was under control in 10 patients $(22.7 \%)$ and uncontrolled in 6 patients $(13.6 \%)$. The remaining patients $(28 / 44,63.6 \%)$ were newly diagnosed. At the time of SRS, metastases to organs other than the brain were present in 19 patients (43.2\%). Brain metastases were located in the subtentorial area in 10 patients $(22.7 \%$ ) and in both the supratentorial and subtentorial area in 34 patients $(77.3 \%)$. Hemorrhage was present in 9 patients (20.5\%), and hydrocephalus in 7 (15.9\%). The median number of tumors was 4 (range, 1-24). The median diameter and volume of the metastases were $3.5 \mathrm{~cm}$ (range, $2.1-5.7 \mathrm{~cm}$ ) and $12.5 \mathrm{cc}$ (range, 1.8-39.7 cc), respectively.
The median volume of the peritumoral edema was $36.3 \mathrm{cc}$ (range, 3.7-100.3 cc). The median ratio of tumor volume to cerebellum volume was $8.7 \%$ (range, $1.3-27.0 \%$ ). The median ratio of peritumoral edema volume to cerebellum volume was $25.0 \%$ (range, $2.5-68.6 \%$ ).

\section{PFS and survival}

Of the 44 patients, 2 worsened following SRS. One patient underwent emergency life-saving surgery and was alive at the time of writing. The other patient was accepted for palliative care and died three months later. In the remaining 42 patients, 21 patients achieved the optimal effect of partial response, and 21 patients achieved stable disease during the followup period. The 6- and 12-month PFS rates were $68.2 \%$ and $31.8 \%$ respectively with median neurological PFS of 9.0 months (range, 1-32 months) as shown in Figure 1. None of the variables tested was predictive of local PFS (Table 2).

At the completion of the study, 11 patients (25\%) were alive and 33 patients (75\%) deceased. Death occurred in 9 patients $(27.3 \%)$ as a result of neurological progression, whilst $24(72.7 \%)$ patients died due to progression of extracranial disease. The OS rate at 6 and 12 months was $79.5 \%$ and $43.2 \%$, respectively (Figure 2). Female, central ataxia, high GPA score, metastases only located in subtentorial area (Figure $3 A$ ), number of metastases $<5$, systemic therapy after SRS (Figure $3 B$ ) were significant on univariate analysis (Table 2). Metastases only located in subtentorial area (hazard ratio, 4.515; $95 \%$ confidence interval, 1.297-15.719; $\mathrm{P}=0.018$ ) and systemic therapy after SRS (hazard ratio, 0.227; 95\% confidence interval, 0.074 $0.703 ; \mathrm{P}=0.010$ ) were significant risk factors associated with survival in multivariate analysis. Tumor diameter, tumor volume and peritumoral edema volume were not significant with survival in univariate analysis (Table 2).

\section{Neurological and functional outcomes}

Focal neurologic deficits such as dizziness, headache, nausea and vomiting, central ataxia, motor weakness, dysarthria, epilepsy and visual dysfunction, improved in most of the patients (Table 3). Two patients reported worsened dizziness, nausea and vomiting after SRS treatment, whilst one reported an increase in headaches. The KPS score improved with a mean pre-treatment KPS score of 72.05 (median, 70; range, 50-90) vs. a mean KPS score of 74.77 (median, 75; range, 50-100) after treatment. 
Table 1 Summary of baseline patient characteristics

\begin{tabular}{|c|c|}
\hline Characteristics & No. (\%) \\
\hline \multicolumn{2}{|l|}{ Age } \\
\hline$\leq 50$ & $14(31.8)$ \\
\hline$>50$ & $30(68.2)$ \\
\hline \multicolumn{2}{|l|}{ Gender } \\
\hline Male & $29(65.9)$ \\
\hline Female & $15(34.1)$ \\
\hline \multicolumn{2}{|l|}{ Symptom } \\
\hline Yes & $43(97.7)$ \\
\hline No & $1(2.3)$ \\
\hline \multicolumn{2}{|l|}{ Smoking } \\
\hline Yes & $20(45.5)$ \\
\hline No & $24(54.5)$ \\
\hline \multicolumn{2}{|l|}{ KPS score } \\
\hline$\geq 70$ & $35(79.5)$ \\
\hline$<70$ & $9(20.5)$ \\
\hline \multicolumn{2}{|l|}{ RTOG-RPA class } \\
\hline I & $5(11.4)$ \\
\hline II & $32(72.7)$ \\
\hline III & 7 (15.9) \\
\hline \multicolumn{2}{|l|}{ GPA score } \\
\hline$\leq 1$ & $10(22.7)$ \\
\hline $1.5-2.5$ & $21(47.7)$ \\
\hline$\geq 3$ & $13(29.5)$ \\
\hline \multicolumn{2}{|l|}{ Pathology } \\
\hline Adenocarcinoma & $26(59.1)$ \\
\hline Squamous carcinoma & $4(9.1)$ \\
\hline Others & $14(31.8)$ \\
\hline \multicolumn{2}{|l|}{ Status of primary cancer } \\
\hline Controlled & $10(22.7)$ \\
\hline Uncontrolled & $6(13.6)$ \\
\hline Newly diagnosed & $28(63.6)$ \\
\hline \multicolumn{2}{|l|}{ Extracranial metastases } \\
\hline Present & $19(43.2)$ \\
\hline Absent & $25(56.8)$ \\
\hline
\end{tabular}

Table 1 (continued)
Table 1 (continued)

\begin{tabular}{lc}
\hline Characteristics & No. (\%) \\
\hline Location of brain metastases & $34(77.3)$ \\
Supratentorial \& subtentorial & $10(22.7)$ \\
Subtentorial & \\
Hemorrhage & $9(20.5)$ \\
Yes & $35(77.8)$ \\
No & \\
Hydrocephalus & $7(15.9)$ \\
Yes & $37(84.1)$ \\
No & \\
Number of brain metastases & 31 (70.5) \\
$\geq 5$ & $13(29.5)$ \\
$<5$ & $3.5(2.1-5.7)$ \\
Tumor diameter (cm), median (range) & $12.5(1.8-39.7)$ \\
Tumor volume (cc), median (range) & $36.3(3.7-100.3)$ \\
Edema volume (cc), median (range) & $143.6(109.2-176.0)$ \\
Cerebellum volume (cc), median (range) & \\
\hline KPS, Karnofsky performance status; RTOG-RPA, Radiation \\
graded prognostic assessment.
\end{tabular}

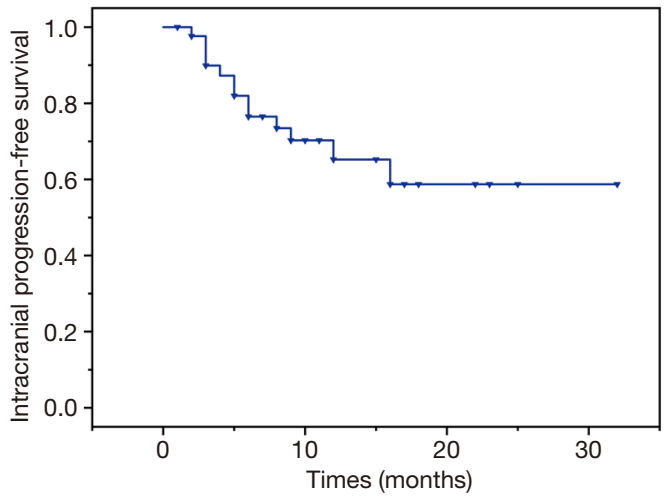

Figure 1 Kaplan-Meier curve showing intracranial progressionfree survival in 44 patients with large cerebellum metastases.

\section{Toxicity}

On the basis of the Common Terminology Criteria for Adverse Events by RTOG, acute toxicities were observed in 20 patients (grade 1 in 17 patients, grade 2 in 2 patients, grade 3 in 1 patient). Radiation necrosis was not observed 
Table 2 Pre-SRS clinical symptoms and post-SRS outcomes

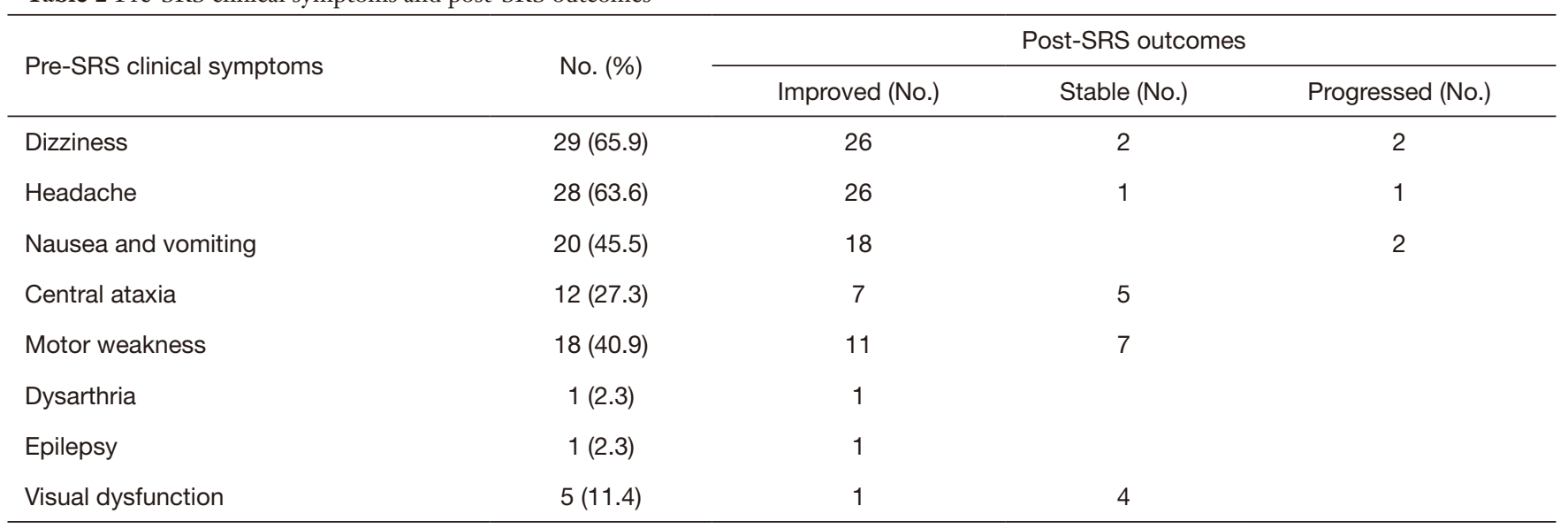

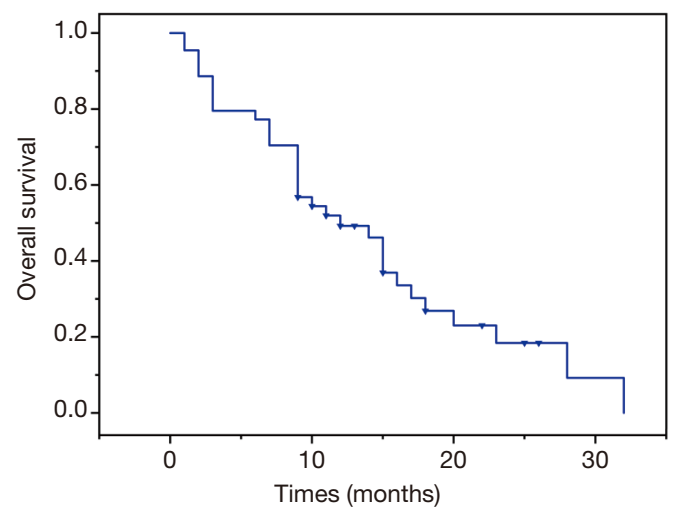

Figure 2 Kaplan-Meier curve showing survival in 44 patients with large cerebellum metastases.

during the follow-up period.

\section{Discussion}

SRS is an effective option in the treatment of large brain metastases, especially in radiosensitive cancers. The National Comprehensive Cancer Network (NCCN) guidelines recommend fractionated SRS in the treatment of brain metastases $>3 \mathrm{~cm}$. Large brain metastases are more abundant with hypoxic tumor cells than small brain metastases $(9,10)$. Fractionated administration of the radiation dose is beneficial to reoxygenation and redistribution of the cell cycle, which renders hypoxic tumor cells more radiosensitive. In addition, fractionated SRS minimizes toxicity to late-responding healthy tissue and decreases the incidence of brain necrosis. The suitable dose selection and numbers of sessions for large brain metastases, however, remains controversial. Factors such as tumor locations, size, number, peritumoral edema volume and the performance of patients may influence clinical decision making.

It is generally true that the larger the metastases, the more serious the symptoms produced. However, peritumoral edema, by causing neurological effects, will also influence this, particularly when the oedema area is larger than that of the tumor. This study revealed tumor and peritumoral edema volumes of between $1.8-39.7 \mathrm{cc}$, and 3.7-100.3 cc respectively. The median volume of the peritumoral edema seen accounts for $25 \%$ of that of the posterior fossa. In this limited space, the presence of large tumors and edema may result in cerebral hernia, oppressing the brainstem and causing respiratory and cardiac arrest.

The results of this study demonstrate that SRS is safe and effectively relieves the symptoms of large cerebellum metastases from lung cancer.

With the exception of two patients, all others achieved remission of their symptoms. This indicates that tumor volume reduction alleviating brain edema contributes to symptomatic improvement (11). However, both tumor volume and peritumoral edema volume did not significantly influence survival.

Although surgical resection has been considered an effective choice for large brain metastases, SRS is an alternative treatment for patients who have progressive cancers. Whilst symptoms can be quickly improved by surgical resection when the tumor is located in a nonfunctional area, when in a functional area, post-operative neurological deterioration may occur. In such cases postoperative SRS is recommended following resection (12).

SRS can relieve neurological symptoms and systemic 
A

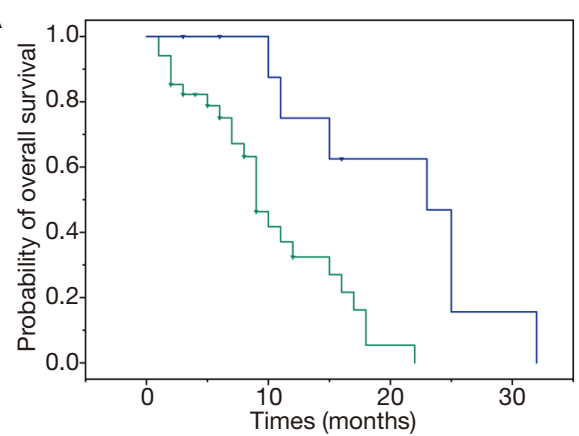

B

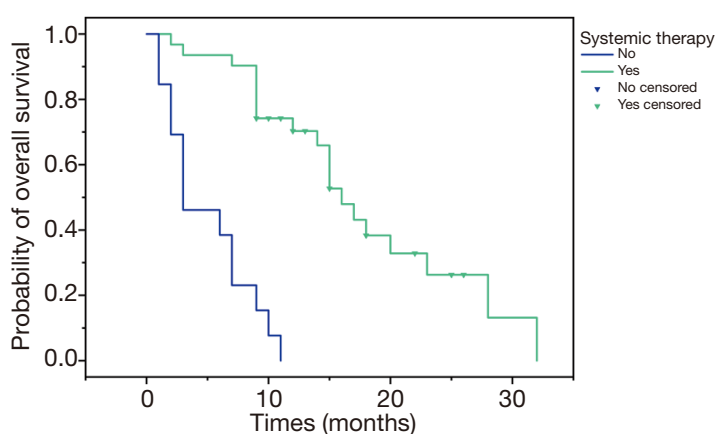

Figure 3 Overall survival (OS) in relation to: (A) location of brain metastases; (B) systemic therapy after SRS. SRS, stereotactic radiosurgery.

Table 3 Prognostic factors for overall survival and progression-free survival (log-rank test)

\begin{tabular}{|c|c|c|c|c|}
\hline \multirow{2}{*}{ Factor } & \multicolumn{2}{|l|}{ OS } & \multicolumn{2}{|l|}{ PFS } \\
\hline & Hazard ratio $(95 \% \mathrm{Cl})$ & $P$ value & Hazard ratio $(95 \% \mathrm{Cl})$ & $P$ value \\
\hline \multicolumn{5}{|l|}{ Age } \\
\hline \multicolumn{5}{|l|}{$\leq 50$} \\
\hline$>50$ & $1.432(0.659-3.112)$ & 0.365 & $1.076(0.331-3.499)$ & 0.903 \\
\hline \multicolumn{5}{|l|}{ Gender } \\
\hline \multicolumn{5}{|l|}{ Male } \\
\hline Female & $0.349(0.150-0.812)$ & 0.015 & $1.668(0.509-5.465)$ & 0.398 \\
\hline \multicolumn{5}{|c|}{ Central ataxia } \\
\hline \multicolumn{5}{|l|}{ Yes } \\
\hline No & $2.250(1.041-4.864)$ & 0.039 & $1.024(0.280-3.753)$ & 0.971 \\
\hline \multicolumn{5}{|l|}{ Smoking } \\
\hline \multicolumn{5}{|l|}{ Yes } \\
\hline No & $1.571(0.766-3.219)$ & 0.218 & $0.358(0.098-1.312)$ & 0.121 \\
\hline \multicolumn{5}{|c|}{ KPS score } \\
\hline \multicolumn{5}{|l|}{$\geq 70$} \\
\hline$<70$ & $1.731(0.770-3.890)$ & 0.184 & $0.849(0.188-3.832)$ & 0.831 \\
\hline \multicolumn{5}{|c|}{ RTOG-RPA class } \\
\hline \multicolumn{5}{|l|}{ I } \\
\hline \multicolumn{5}{|l|}{ II } \\
\hline III & $1.435(0.752-2.740)$ & 0.273 & $0.774(0.289-2.068)$ & 0.609 \\
\hline \multicolumn{5}{|c|}{ GPA score } \\
\hline \multicolumn{5}{|l|}{$\leq 1$} \\
\hline \multicolumn{5}{|l|}{$1.5-2.5$} \\
\hline$\geq 3$ & $0.405(0.243-0.677)$ & 0.001 & $0.807(0.365-1.784)$ & 0.596 \\
\hline
\end{tabular}

Table 3 (continued) 
Table 3 (continued)

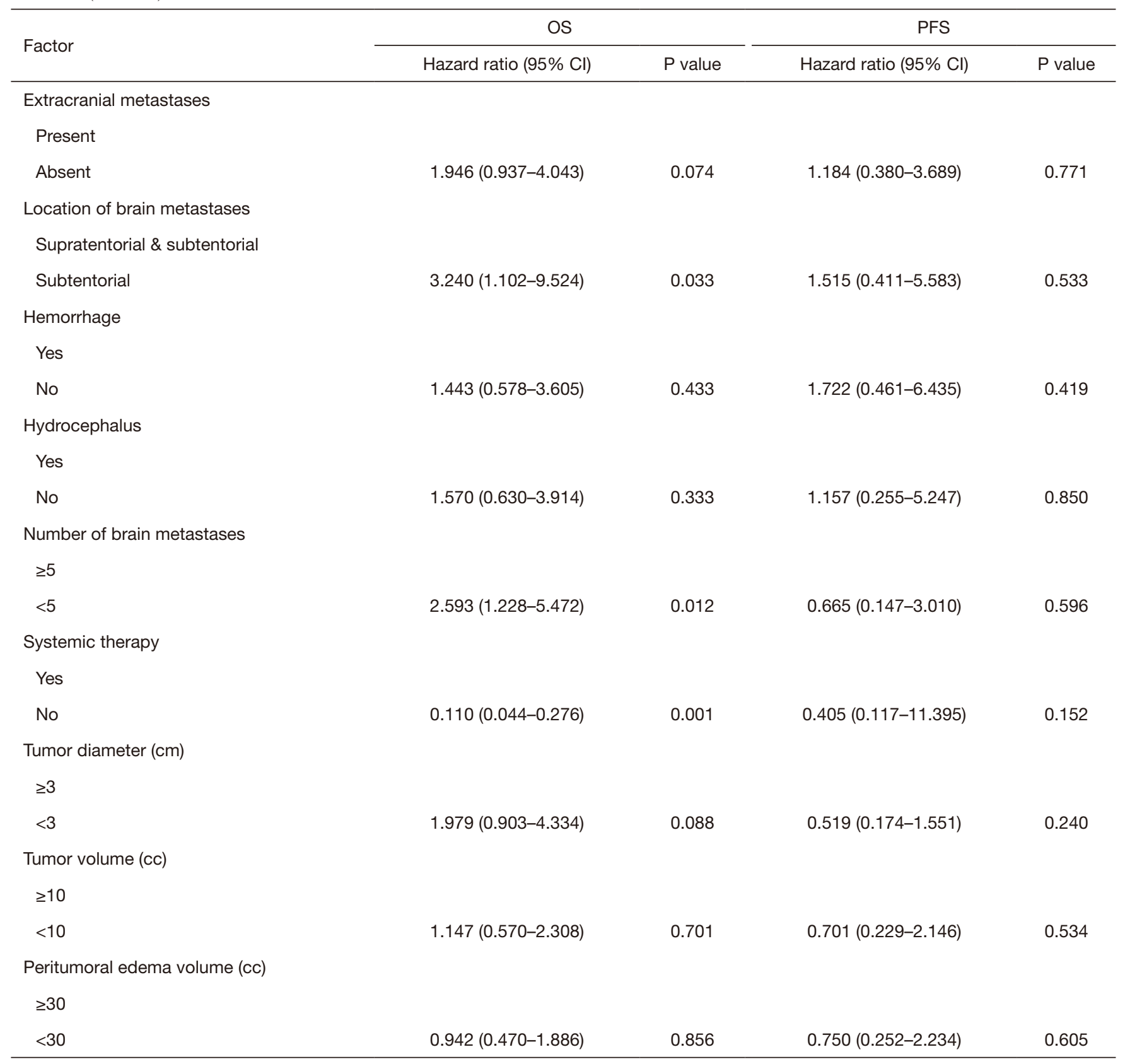

KPS, Karnofsky performance status; RTOG-RPA, Radiation Therapy Oncology Group-recursive partitioning analysis; GPA, graded prognostic assessment.

treatment can prolong the survival of patients with large cerebellum metastases. In this study, $27 \%$ patients died from cranial disease progression and $73 \%$ patients from extracranial disease progression. Systemic treatment following SRS was not provided in $29.5 \%$ of the deceased patients thus the results of local therapy to the brain may be influenced by extracranial disease progression. Systemic treatment for primary sites appears to be essential.

\section{Limitation}

The current study is a retrospective study with limited 
sample size. The biases of patient selection, treatment and clinical institution selection exist.

\section{Conclusions}

The present results confirm those of our earlier study. Tumor volume and peritumoral edema volume cause neurological deficits. SRS for large cerebellum metastases in lung cancer patients provides effective neurological palliation with a low incidence of severe toxic effects.

\section{Acknowledgments}

Funding: None.

\section{Footnote}

Reporting Checklist: The authors have completed the STROBE reporting checklist. Available at http://dx.doi. org/10.21037/apm-20-2237

Data Sharing Statement: Available at http://dx.doi. org/10.21037/apm-20-2237

Conflicts of Interest: All authors have completed the ICMJE uniform disclosure form (available at http://dx.doi. org/10.21037/apm-20-2237). The authors have no conflicts of interest to declare.

Ethical Statement: The authors are accountable for all aspects of the work in ensuring that questions related to the accuracy or integrity of any part of the work are appropriately investigated and resolved. The study was approved by IRB of Guangdong Sanjiu Brain Hospital (No. 2020-020-083) and written informed consent was obtained from all patients. All procedures performed in this study involving human participants were in accordance with the Declaration of Helsinki (as revised in 2013).

Open Access Statement: This is an Open Access article distributed in accordance with the Creative Commons Attribution-NonCommercial-NoDerivs 4.0 International License (CC BY-NC-ND 4.0), which permits the noncommercial replication and distribution of the article with the strict proviso that no changes or edits are made and the original work is properly cited (including links to both the formal publication through the relevant DOI and the license).
See: https://creativecommons.org/licenses/by-nc-nd/4.0/.

\section{References}

1. Park K, Kim JW, Chung HT, et al. Single-Session versus Multisession Gamma Knife Radiosurgery for Large Brain Metastases from Non-Small Cell Lung Cancer: A Retrospective Analysis. Stereotact Funct Neurosurg 2019;97:94-100.

2. Lehrer EJ, Peterson JL, Zaorsky NG, et al. Single versus Multifraction Stereotactic Radiosurgery for Large Brain Metastases: An International Metaanalysis of 24 Trials. Int J Radiat Oncol Biol Phys 2019;103:618-30.

3. Lovo EE, Torres LB, Campos FJ, et al. Two-session Radiosurgery as Initial Treatment for Newly Diagnosed Large, Symptomatic Brain Metastases from Breast and Lung Histology. Cureus 2019;11:e5472.

4. Yamamoto M, Higuchi Y, Serizawa T, et al. Three-stage Gamma Knife treatment for metastatic brain tumors larger than $10 \mathrm{~cm} 3$ : a 2-institute study including re-analyses of earlier results using competing risk analysis. J Neurosurg 2018;129:77-85.

5. Zindler JD, Schiffelers J, Lambin P, et al. Improved effectiveness of stereotactic radiosurgery in large brain metastases by individualized isotoxic dose prescription: an in silico study. Strahlentherapie und Onkologie 2018;194:560-9.

6. Yomo S, Hayashi M, Nicholson C. A prospective pilot study of two-session Gamma Knife surgery for large metastatic brain tumors. J Neurooncol 2012;109:159-65.

7. Higuchi Y, Serizawa T, Nagano O, et al. Three-staged stereotactic radiotherapy without whole brain irradiation for large metastatic brain tumors. Int J Radiat Oncol Biol Phys 2009;74:1543-8.

8. Eisele SC, Wen PY, Lee EQ. Assessment of Brain Tumor Response: RANO and Its Offspring. Curr Treat Options Oncol 2016;17:35.

9. Shibamoto Y, Yukawa Y, Tsutsui K, et al. Variation in the hypoxic fraction among mouse tumors of different types, sizes, and sites. Jpn J Cancer Res 1986;77:908-15.

10. Rampling R, Cruickshank G, Lewis A, et al. Direct measurement of $\mathrm{pO} 2$ distribution and bioreductive enzymes in human malignant brain tumors. Int J Radiat Oncol Biol Phys 1994;29:427-31.

11. Hasegawa T, Kato T, Yamamoto T, et al. Multisession gamma knife surgery for large brain metastases. J 
Neurooncol 2017;131:517-24.

12. Ling DC, Vargo JA, Wegner RE, et al. Postoperative stereotactic radiosurgery to the resection cavity for large brain metastases: clinical outcomes, predictors of intracranial failure, and implications for optimal patient

Cite this article as: Lai M, Li S, Zhou J, Zhen J, Li J, Hu Q, Shan C, Ai R, Hong W, Wang H, Ye M, Yang Y, Xiao X, Wen L, Zhou Z, Zhou C, Cai L. Stereotactic radiosurgery for treatment of large cerebellum metastases from lung cancer. Ann Palliat Med 2021;10(1):220-228. doi: 10.21037/apm-20-2237 selection. Neurosurgery 2015;76:150-6; discussion 156-7; quiz 157.

(English Language Editor: B. Draper) 